\title{
Genética, medio ambiente e hipertensión arterial, sumando puntos
}

\section{Genetics, environment and hypertension, adding points}

\author{
Dora Inés Molina* y Germán Camilo Giraldo
}

Asociación Institución Prestadora de Salud Médicos Internistas de Caldas, Universidad de Caldas, Manizales, Colombia

Recibido el 15 de octubre de 2014; aceptado el 17 de octubre de 2014

Disponible en Internet el 6 de diciembre de 2014

\section{Introducción}

El número de personas con enfermedad cardiovascular prevalente conocida en todo el mundo, probablemente supera los 100 millones y afecta a hombres y mujeres, con una relación directamente proporcional con la edad. De todos los estratos socio-económicos, el impacto más analizado pertenece a los países con altos ingresos, pero un $75 \%$ de la carga de las enfermedades cardiovasculares recae en los países de ingresos bajos y medianos como Colombia ${ }^{1}$. La hipertensión sigue siendo uno de los más importantes contribuyentes prevenibles a la enfermedad y muerte cardiovascular. Existe abundante evidencia de ensayos controlados aleatorizados que han demostrado el beneficio del tratamiento antihipertensivo reflejado en la mejoría de los desenlaces posteriores en este grupo de pacientes ${ }^{2}$.

No obstante, hay un nuevo giro y objetivo en el análisis de los pacientes con hipertensión arterial que va de la mano con la capitalización e industrialización creciente de las comunidades, y es el impacto de los hábitos de vida y las características propias de una población sobre la apari-

Véase contenido relacionado en DOI:

http://dx.doi.org/10.1016/j.rccar.2014.05.009

* Autor para correspondencia.

Correo electrónico: doraines56@gmail.com (D.I. Molina). ción temprana de hipertensión arterial, así que tener control sobre la presión arterial y la prevención de la morbimortalidad de la misma, son objetivos claramente alcanzables. Cada vez hay más certeza sobre la posibilidad de que la predisposición genética asociada a determinados grupos étnicos, podría interactuar con factores ambientales para explicar el aumento crítico de la hipertensión en países como el nuestro ${ }^{3}$.

En el artículo de Arango et al., publicado en este número de la Revista, se recalcan los diversos factores que, se ha demostrado, contribuyen a la enfermedad hipertensiva y cardiovascular: inactividad física, sedentarismo, sobrepeso, obesidad abdominal y baja capacidad cardiorrespiratoria, que pueden iniciarse desde la infancia y mantenerse hasta la adultez. Los resultados, no dejan de sorprender. Nuestros niños y adolescentes, permanecen gran parte del tiempo sentados viendo televisión, perpetuando un círculo vicioso de sedentarismo, sobrepeso y obesidad, que evidencia el incremento de aparición de hipertensión arterial a edades tempranas, reportado en la literatura ${ }^{4}$. Estos hallazgos en niños colombianos, enriquecen la literatura a fin de avalar la necesidad de intervenciones tempranas para prevenir no solo los conglomerados sino la aparición de eventos adversos y desenlaces cardiovasculares futuros. Una de las grandes fortalezas de este estudio, es corroborar cómo desde la infancia la inactividad física adquiere protagonismo en la génesis de la hipertensión, asociado con la 
adiposidad, como lo reportan diversas publicaciones, de ahí que se cuente con herramientas sencillas en el consultorio que permiten visualizar aquellos niños en quienes han comenzado a incrementarse las cifras de tensión arterial. Cabe resaltar el análisis de la epidemiología local y cómo los resultados difieren poco con lo encontrado en el mundo y en especial en Europa. Los autores, además, son precisos y coherentes con las limitaciones que reconocen.

Los antecedentes familiares de hipertensión arterial están presentes hasta en el 70 y $80 \%$ de todos los pacientes con hipertensión primaria, que no tienen etiología subyacente identificable, y en aproximadamente un $50 \%$ de los niños hipertensos. En la población infantil, la hipertensión primaria se da como resultado de la interacción de múltiples genes y factores ambientales. Se ha estimado que los factores genéticos representan aproximadamente el $30 \%$ de la variación de la presión arterial en diversas poblaciones ${ }^{5}$, hecho que nos conduce a pensar que si el conglomerado entre las variables anteriores incrementa la presión arterial, no se deben olvidar otros elementos que parecen tener protagonismo en esta población, quizás un factor para analizar en estudios posteriores en nuestra población.

De otra parte, existe evidencia de que la lactancia materna puede estar asociada con niveles inferiores de presión arterial en la infancia. La reducción de las presiones sistólica y diastólica es mayor en niños que fueron amamantados de forma exclusiva, y la reducción de la presión arterial sistólica se prolonga de manera directamente proporcional con la duración de la lactancia materna ${ }^{6}$.

De igual forma, se cuenta con mayor evidencia en cuanto a que los factores prenatales y neonatales contribuyen a elevar la presión arterial. Hay datos que demuestran el papel preponderante del bajo peso al nacer en el desarrollo de hipertensión arterial primaria. Además, la exposición intrauterina a la preeclampsia se asocia con aumento de las presiones sistólica (media 2,4 $\mathrm{mm} \mathrm{Hg}$ ) y diastólica (media $1,4 \mathrm{~mm} \mathrm{Hg}$ ), así como del índice de masa corporal (media $0,62 \mathrm{~kg} / \mathrm{m}^{2}$ ) de los neonatos ${ }^{7}$.

Bien se sabe que en Latinoamérica, la desnutrición materna e infantil ha sido un problema irresoluto en un porcentaje considerable de las poblaciones y que se ha encontrado una alta prevalencia de hipertensión arterial en niños, adolescentes y adultos con mal estado nutricional. Diversos estudios en animales han demostrado que una mala nutrición durante el embarazo conlleva hijos con niveles altos de presión arterial ${ }^{8}$. En Bucaramanga, un estudio realizado por López-Jaramillo et al. ${ }^{3}$, evaluó jóvenes entre los 10 y 13 años, en quienes además de tomar medidas antropométricas, evaluaron fuerza de agarre, aptitud física, composición corporal, entre otros, y encontraron una relación inversamente proporcional entre la presión arterial y el índice HOMA (del inglés homoeostasis model assessment) ( $p=0,001$ para todos) con la fuerza de agarre y la aptitud física?. A todos estos factores deben sumársele los resultados de estudios internacionales que demuestran cómo a medida que los países tienen menores ingresos, poseen peores desenlaces cardiovasculares y medidas de intervención efectivas $^{10}$.

Vemos entonces, que quizás en nuestra práctica diaria podemos realizar un análisis más global, las variables pueden ser incontables, y su suma va llevando a que finalmente en la adultez el paciente comience con un evento cerebrovascular catastrófico o un evento coronario agudo que lo lleva a discapacidad, falla cardiaca y muerte.

Existen mecanismos de implementación factible desde la asesoría preconcepcional, para pensar en el futuro niño cuya enfermedad se manifestará en el adulto. Los ensayos clínicos y la práctica diaria demuestran que podemos prevenir estos desenlaces con educación, intervenciones apropiadas y conocimiento sobre aquellos que están en riesgo.

Recomendamos al lector vigilar con detalle la tendencia epidemiológica en su medio y aprovechar estas herramientas de consultorio para iniciar de manera temprana recomendaciones de hábitos de vida saludable para que las conductas que el paciente aprende de su mayor, tengan impacto en algún momento de su existencia.

\section{Bibliografía}

1. Yusuf S, Islam S, Chow CK, Rangarajan S, Dagenais G, Diaz $R$, et al. Use of secondary prevention drugs for cardiovascular disease in the community in high-income, middle-income, and low-income countries (the PURE Study): a prospective epidemiological survey. Lancet. 2011;378(9798): 1231-43.

2. James PA, Oparil S, Carter BL, Cushman WC, DennisonHimmelfarb C, Handler J, et al. 2014 evidence-based guideline for the management of high blood pressure in adults: report from the panel members appointed to the Eighth Joint National Committee (JNC 8). JAMA. 2014;311(5):507-20.

3. López-Jaramillo P, Velandia-Carrillo C, Álvarez-Camacho J, Cohen DD, Sánchez-Solano T, Castillo-López G. Inflammation and hypertension: are there regional differences? Int J Hypertens. 2013;2013:492-4.

4. Tu W, Eckert GJ, DiMeglio LA, Yu Z, Jung J, Pratt JH. Intensified effect of adiposity on blood pressure in overweight and obese children. Hypertension. 2011;58:818.

5. Hunt SC, Ellison RC, Atwood LD, Pankow JS, Province MA, Leppert MF. Genome scans for blood pressure and hypertension: the National Heart Lung, and Blood Institute Family Heart Study. Hypertension. 2002;40:1

6. Martin RM, Ness AR, Gunnell D, Emmett P, Davey Smith G, ALSPAC Study Team. Does breast-feeding in infancy lower blood pressure in childhood? The Avon Longitudinal Study of Parents and Children (ALSPAC). Circulation. 2004;109:1259.

7. Davis EF, Lazdam M, Lewandowski AJ, Worton SA, Kelly B, Kenworthy $\mathrm{Y}$, et al. Cardiovascular risk factors in children and young adults born to preeclamptic pregnancies: a systematic review. Pediatrics. 2012;129:e1552.

8. López-Jaramillo P, Camacho PA, Forero-Naranjo L. The role of environment and epigenetics in hypertension. Expert Rev CardiovascTher. 2013;11(11):1455-7.

9. Camacho PA, Pinzon S, Hormiga C, Trejos-Suarez J, Duperly J, López-Jaramillo P. Low muscle strength is associated with metabolic risk factors in Colombian children: the ACFIES study. PLoS One. 2014;9(4):e93150.

10. Szuba A, Li W, Yusoff K, Yusufali A, Rangarajan S, Teo K, et al. Prospective Urban Rural Epidemiology (PURE) study: Baseline characteristics of the household sample and comparative analyses with national data in 17 countries. Am Heart J. 2013;166(4):636-46. 